W stużbie tradycji i odnowy liturgicznej. 50 lat Instytutu Liturgicznego w Krakowie (1968-2018), red. P. Nowakowski, J. Mieczkowski, Kraków 2019, s. 301-316.

ISBN 978-83-7438-849-8 (wersja drukowana), ISBN 978-83-7438-850-4 (wersja online) DOI:http://dx.doi.org/10.15633/9788374388504.23

André Lossky

Instytut Teologit Prawoseawnej Świętego Sergiusza w Paryżu, Francja

\title{
Les Semaines d'études liturgiques Saint-Serge de Paris, aide possible à un renouveau liturgique
}

Depuis près de deux siècles, les questions liturgiques ont fait et font toujours l'objet d'importantes études et recherches. Les membres conscients des Églises s'attachent à assurer une liturgie qui continue d'actualiser les Mystères divins chrétiens, comme l'évoque, par exemple, la voyageuse Égérie au 4 siècle à Jérusalem. Décrivant entre autres la lecture dominicale, dans l'Évangile, du « récit de la Résurrection du Seigneur » qui a " tant souffert pour nous ", elle mentionne des réactions de l'assemblée, utilisant pour cela des expressions suggérant une forte émotion des participants qui ne peuvent rester indifférents aux événements salvateurs (Itinéraire, 24, 10). L'action liturgique doit s'efforcer de faire participer concrètement chaque assemblée, et chacun de ses membres, aux bienfaits de l'œuvre de salut procurée par Dieu. Ainsi peut être énoncée la fonction première de toute liturgie, qui sans cela tendrait à se figer vers des formes observées pour elles-mêmes, dépourvues de contenu théologique. Éviter une telle déviance : tel nous paraît l'enjeu des travaux menés par les chercheurs d'un renouveau liturgique, inséparable d'une prise de conscience théologique.

Notre exposé rappellera d'abord brièvement les débuts de l'Institut de Théologie orthodoxe Saint-Serge, fondé à Paris en 1925, puis soulignera 
la spécificité du lieu en matière de liturgie, pour tenter enfin de cerner l'esprit dans lequel s'y déroulent, depuis maintenant près de 65 ans, les Semaines d'Études liturgiques annuelles. Ces rencontres à la fois œcuméniques et académiques cherchent à promouvoir une meilleure connaissance des faits liturgiques d'hier et d'aujourd'hui, pour contribuer autant que possible à ce renouveau toujours recherché ${ }^{1^{*}}$.

\section{Introduction : 1'Institut de Théologie orthodoxe Saint-Serge de Paris}

Des émigrés russes sont arrivés en grand nombre au début du $20^{\mathrm{e}}$ siècle en Europe occidentale et à Paris. Beaucoup d'entre eux, chrétiens orthodoxes, ont pris conscience de besoins ressentis au sein de leur communauté, besoins à la fois pastoraux, académiques et pédagogiques. Pour y répondre, ils ont décidé de fonder un lieu de prière, de réflexion et de formation en théologie. Ainsi fut créé dans un quartier du $19^{\mathrm{e}}$ arrondissement, au nord-est de Paris, un lieu de culte et en même temps d'enseignement théologique, les deux placés conjointement sous la protection de Saint Serge, abbé de Radonège, fondateur au $14^{\mathrm{e}}$ siècle d'un monastère près de Moscou, dédié à la Sainte Trinité ${ }^{2}$.

La constitution en France d'un lieu ecclésial de formation théologique a été vivement souhaitée par le Métropolite Euloge, (Georgievsky, m. 1946), devenu à partir de 1921 évêque diocésain des orthodoxes arrivés de Russie en France. Cette fondation s'inscrit dans une tradition déjà observée en Russie, où ont fonctionné des académies de théologie et des séminaires

1 * Je remercie chaleureusement, pour leurs conseils et leur patiente relecture, M. Adrian Craciun et les Pères Gilles Drouin et Marcel Metzger.

2 Sur le choix de Saint Serge comme protecteur du lieu, voir Le chemin de ma vie. Mémoires du Métropolite Euloge (Traduit du russe par le père Pierre Tchesnakoff), Paris 2005 (original paru en 1947), p. 363-365.

Sur l'importance théologique de la dévotion de Saint Serge à la Sainte Trinité, voir par exemple L. Ouspenski, La Théologie de l'icône dans l'Église orthodoxe, Paris 1980, p. 227 ; voir aussi Vie de Saint Serge, ch. 7-8 et 32, citée par P. Gonneau, À l'aube de la Russie moscovite. Serge de Radonège et André Roublev. Légendes et images (XIVe-XVIIe siècles), Paris 2007, p. 93-99, 92-93. 
ecclésiastiques. Ces activités se sont poursuivies hors de Russie, et particulièrement à Paris, malgré les difficultés, grâce à un fort investissement des émigrés récents. Parmi eux figuraient de grands intellectuels, penseurs, enseignants, et aussi des ecclésiastiques et des théologiens, porteurs d'un message au sein duquel les questions liturgiques n'occupent pas la dernière place. Par les célébrations liturgiques aussi bien que par des activités telles qu'enseignement ou conférences, ce témoignage est proposé aujourd'hui aussi à toute personne intéressée par ces questions³.

\section{Le rôle liturgique de la colline Saint-Serge}

Le lieu est situé sur une propriété parisienne où avait été construit vers 1850 un temple protestant, mis ensuite en vente comme dommage de guerre et acheté par la communauté orthodoxe sur l'instigation du Métropolite Euloge. Dans l'église aménagée depuis 1925 selon la tradition byzantine, des célébrations paroissiales sont organisées les dimanches et fêtes. C'est aussi un des lieux à Paris où est célébré quotidiennement l'office divin orthodoxe, en l'occurrence principalement Vêpres et Matines.

\subsection{Prière, théologie et formation liturgique}

Cette célébration fréquente crée une émulation à la fois liturgique et spirituelle. En sa forme académique et intellectuelle, la théologie n'est en effet qu'un aspect particulier de la démarche profonde de toute personne chrétienne, à savoir la quête, avant tout par la prière liturgique et personnelle, d'une rencontre avec Dieu.

3 On trouvera plus de détails sur la formation théologique ancienne en Russie et sur ses débuts à Paris dans: P. A. Kniazeff, L'institut Saint-Serge. De l'académie d'autrefois au rayonnement d'aujourd'hui, Paris 1974 (Le point théologique, 14), ch. 1 et 2 ; voir aussi les mémoires du Métropolite Euloge ; cet ouvrage (voir note précédente) présente notamment la situation intellectuelle religieuse en Russie au $19^{\mathrm{e}}$ siècle, P. A. Kniazeff, L'institut Saint-Serge, p. 63-106 ; l'auteur expose aussi les motivations l'ayant poussé à favoriser l'ouverture de la Paroisse et de l'Institut Saint-Serge à Paris, au sein du diocèse dont il avait la charge. P. A. Kniazeff, L'institut Saint-Serge, p. 363 . 
Loin d'un devoir dont le croyant s'acquitterait au risque de le bâcler, la liturgie chrétienne constitue la partie visible de cette rencontre avec Dieu, exprimée et encouragée avec insistance par les textes liturgiques proclamés ou chantés lors des célébrations. Ce rythme liturgique, auquel chacun est invité à participer selon sa disponibilité et ses forces, constitue aussi pour les étudiants, les enseignants et les chercheurs une occasion concrète d'apprentissage pratique de l'utilisation des livres liturgiques et de la sanctification du temps, selon les différents cycles tels que les observe le rite byzantin ${ }^{4}$.

L'enracinement liturgique est en outre une nourriture qui alimente la réflexion théologique et contribue à faire assimiler l'enseignement dispensé dans un Institut de Théologie. Celui-ci est organisé à Saint-Serge selon les différentes disciplines académiques, telles que sciences et théologie bibliques, pensée patristique, histoire des Églises, théologie des dogmes, histoire des conciles, traditions canoniques,... Dans cette énumération non exhaustive, la théologie liturgique est aussi objet d'un enseignement non réduit à une simple description de la structure ancienne ou actuelle des célébrations, mais cette notion a mûri peu à peu depuis une science vers une théologie liturgique, à proprement parler, cette dernière devant prendre en compte la première, selon une tradition d'interprétation qui à Saint-Serge n'est pas restée figées.

\subsection{Engagement oecuménique}

À côté de la recherche et de l'enseignement, les membres du corps professoral de l'Institut Saint-Serge, ainsi que certains étudiants avancés, sont engagés dans divers groupes de dialogue et de rencontres avec des personnes appartenant à d'autres confessions chrétiennes; des conférences

4 Sur les cycles et les livres liturgiques byzantins, voir J. Getcha, Le Typicon décrypté. Manuel de liturgie byzantine, Paris 2009, p. 15-52, sp. 38-40.

5 Cette évolution de l'enseignement liturgique a été caractérisée plus précisément par J. Getcha, Du maître au disciple : la notion de 'théologie liturgique' chez les pères Cyprien Kern et Alexandre Schmemann, dans : La joie du Royaume. Actes du colloque international. L'héritage du Père Alexandre Schmemann, Paris, 11-14 décembre 2008, éd. A. Lossky, C. Sollogoub, D. Struve, Paris 2012, p. 131-147, ici 137-144. 
et des colloques sont aussi organisés régulièrement sur divers thèmes théologiques inter-confessionnels et inter-religieux. L'Institut Saint-Serge de Paris connaît ainsi un certain rayonnement allant au-delà d'un service rendu strictement à des fidèles orthodoxes; ce rayonnement cherche à atteindre aussi d'autres penseurs, chrétiens ou non ${ }^{6}$. Un cas particulier de cette activité envers l'extérieur est constitué par les Semaines d'Études liturgiques annuelles, objet de notre présentation.

\section{Les Semaines d'Études liturgiques Saint-Serge}

Plusieurs évaluations de ces rencontres, ainsi que de l'esprit qui les anime, ont déjà été publiées. Sans les reprendre, on s'attachera ici d'une part à relever quelques points saillants, pour montrer ce qui a peu varié depuis les premières rencontres, et d'autre part à essayer de discerner des évolutions plus récentes, afin que chacun puisse se faire une idée du bien-fondé des choix opérés par les organisateurs, anciens ou actuels.

\subsection{Origines}

L'initiative de ces colloques liturgiques découle entre autres d'un constat: des rencontres œcuméniques organisées dans les années 1950 ont été ressenties par certains de leurs participants comme trop générales et peu productives. Ainsi a mûri un projet de dialogues entre chrétiens non autour de questions divergentes, mais au contraire de ce qui les réunit. La liturgie a été choisie comme un terrain concret, lié à la théologie et utile à étudier pour constater les points communs, partagés par un plus grand nombre, mais également pour signaler des différences pouvant résulter d'évolutions particulières ou de mécompréhensions à dépasser.

Des contacts ont été pris en ce sens en 1952 entre des professeurs de Saint-Serge et des moines du Monastère bénédictin Sainte-Croix à Chevetogne en Belgique ; ces échanges ont donné lieu à la première des Semaines

6 Cette activité est présentée plus en détails par le P. A. Kniazeff, L'institut Saint-Serge. De l'académie..., ch. 3-4, à compléter par une consultation du site électronique de l'Institut : www.saint-serge.net (22.09.2018), onglet « événements ». 
d'études liturgiques Saint-Serge, tenue en juillet 1953. Les origines de ces rencontres académiques ont été bien analysées par le P. Nicolas Egender, moine de Chevetogne, auteur d'une contribution dans un volume ayant marqué la $60^{\mathrm{e}}$ Semaine, tenue en 2013. L'auteur caractérise ainsi les débuts de ces colloques : "Les grandes initiatives sont dues, le plus souvent, à l'audace d'une ou deux personnes animées d'une grande idée »7. Ce projet commun est aussi encouragé par les autorités catholiques romaines, qui favorisent la découverte réciproque, jugée enrichissante, entre les traditions des diverses confessions chrétiennes. Ceci sera confirmé plus tard par le pape Jean-Paul II dans des documents officiels ${ }^{8}$.

\subsection{Méthodologie : des rencontres académiques et oecuméniques}

Les premiers organisateurs des Semaines d'études liturgiques à Saint-Serge ont voulu leur conférer une dimension scientifique et ont invité des chercheurs spécialisés pour qu'ils partagent leurs découvertes. Ce principe scientifique implique, encore aujourd'hui, un travail centré sur une mise au jour et une analyse de sources documentaires. Jusqu'en 1960, les thèmes généraux des colloques étaient libres, mais les organisateurs ont pris ensuite l'habitude, toujours maintenue, de choisir chaque année un domaine liturgique spécifique autour duquel sont regroupées les communications. Ce thème est déterminé après plusieurs délibérations informelles entre organisateurs et participants. La majorité des exposés porte sur des textes, surtout liturgiques, mais incluant aussi

7 N. Egender, Aux origines des Semaines d'études liturgiques de Saint-Serge. L'archimandrite Cyprien Kern, dom Lambert Beauduin et les moines de Chevetogne, dans : 6o Semaines liturgiques à Saint-Serge : bilans et perspectives nouvelles. $60^{\text {e }}$ Semaine d'Études liturgiques. Paris, Institut Saint-Serge, 24-27 juin 2013, éd. A. Lossky, G. Sekulovski, Münster 2016, p. 241267, ici p. 241 (Studia Oecumenica Friburgensia, 71). Voir aussi P. A. Kniazeff, L'institut Saint-Serge. De l'académie..., p. 113, signalant les Semaines comme devenues en 1974 " une institution».

8 Cités par le P. M. Sodi, Les Semaines liturgiques de Saint-Serge (1953-2012) : Thèmes, débats et méthodes. Quel avenir ?, dans : 60 Semaines liturgiques à Saint-Serge..., p. 311-319, ici 315-316. 
quelques analyses soit de vestiges archéologiques ${ }^{9}$, soit de documents iconographiques ${ }^{10}$.

Un tel choix méthodologique dépasse une simple description de coutumes ou d'usages observés ici ou là, ce qui relèverait plutôt d'un témoignage, pour arriver à une véritable analyse critique de pratiques liturgiques attestées par des documents d'hier ou d'aujourd'hui, pour en proposer une interprétation doctrinale. Comme le souligne notamment le P. Alexandre Schmemann, la théologie liturgique se construit à partir de la liturgie elle-même, telle que fixée dans des textes ou documents spécifiques, plutôt que d'obéir à un principe extérieur ou à un a priori qui lui serait artificiellement imposé. Sans être le seul à avancer ce principe, cet auteur souligne avec insistance que la liturgie, avant d'être objet d'analyse, est elle-même source de théologie ${ }^{11}$.

\section{3. Échanges et dialogues}

La plupart des contributions, déjà à l'oral, suscitent des discussions souvent fructueuses, dans un climat de grande confiance réciproque et d'amitié

9 Voir notamment les deux volumes consacrés à l'espace liturgique, reprenant les exposés des $51^{\mathrm{e}}$ et $52^{\mathrm{e}}$ Semaines, tenues en 2004 et en 2005, parues respectivement en 2005 et 2006 ; références dans la récapitulation dressée par P. M. Sodi, Les Semaines liturgiques..., p.313-315; liste bibliographique à compléter par sa présentation en italien : P. M. Sodi, Le'Semaines d'Études Liturgiques', dans : Rites de communion. Conférences Saint-Serge. LVe Semaine d'Études Liturgiques. Paris, 23-26 juin 2008, Città del Vaticano 2010, p. VII-XV, ici IX-XIII (Monumenta Studia Instrumenta Liturgica, 59).

10 Par exemple : exposés des Pères Marcel Metzger dans les colloques tenus en 1985 et 1986, et Nicolas Ozoline en 1977, 1979, 1983 ou 1984 ; références complètes dans une nomenclature dépouillant chaque contribution dans les anciens volumes, jusqu'en 1992 : P. A. Triacca, Le «Conferenze San Sergio ». Settimane ecumeniche di studi liturgici, Rome 1994, index p. 62, renvoyant aux références des volumes.

11 Voir ses remarques: Théologie liturgique. Remarques méthodologiques, dans : La liturgie, son sens, son esprit, sa méthode : liturgie et théologie. Conférences Saint-Serge. 28 e Semaine d'études liturgiques, Paris, 30 juin-3 juillet 1981, Rome 1982, p. 297-303, ici 298 et 301 (Bibliotheca Ephemerides Liturgicae. Subsidia, 27). Cf. A. Schmemann, L'Eucharistie, sacrement du Royaume, Paris 1985, p. 4 : « Le premier principe de la théologie liturgique $[\ldots]$ consiste $[\ldots]$ à ne pas partir 
sans familiarité, état d'esprit que les organisateurs actuels espèrent maintenir au maximum. Ces échanges permettent ensuite aux auteurs d'approfondir leurs recherches pour en faire profiter les lecteurs de la publication qui suit. Cette attitude fraternelle veut aboutir à un non-jugement face à une position avec laquelle un participant pourrait se trouver en éventuel désaccord.

\subsection{Participants, public visé}

Les rencontres anciennes se voulaient réservées à des spécialistes, heureux de se retrouver pour confronter leurs points de vue sur des documents parfois difficiles à interpréter sans un minimum d'initiation. Mais à partir du $63^{\mathrm{e}}$ colloque tenu en $2016^{12}$, les organisateurs ont choisi d'élargir quelque peu le cercle des participants, selon des modalités énoncées plus loin dans cet exposé (infra, § 37).

En plus des auteurs de communications, les Semaines liturgiques rassemblent aussi un public en moins grand nombre que les orateurs, venant profiter des exposés et des échanges pour enrichir leur bagage liturgique et théologique, dans le contexte œcuménique n'ayant pas cessé de caractériser les Semaines liturgiques. Qu'il s'agisse d'orateurs ou d'auditeurs, les participants comptent aussi des pasteurs, des évêques, et plusieurs personnes engagées à un titre ou un autre dans l'action catéchétique, dans l'organisation pratique des célébrations ou dans des dialogues œcuméniques moins centrés sur la liturgie. Cette constatation de diversité découle des questions posées lors des échanges qui suivent les exposés, tout comme des conversations informelles lors des repas ou pauses entre séances.

\subsection{Domaines de recherche}

Les thèmes liturgiques choisis pour chaque année sont très divers : Eucharistie, autres sacrements, théologie trinitaire, ecclésiologie, eschatologie,

de schèmes intellectuels et abstraits superposés à la liturgie, mais à se fonder sur la prière même de l'Église, à savoir : d'abord sur son ordre » (italiques de l'auteur).

12 Parution: Traditions recomposées. Liturgie et doctrine en harmonie ou en tension. $63^{\mathrm{e}}$ Semaine d'Études liturgiques, Paris, Institut Saint-Serge, 21-24 juin 2016, éd. A. Lossky, G. Sekulovski, Münster 2017 (Studia Oecumenica Friburgensia, 80). 
toujours en rapport avec la liturgie. L'orientation des recherches a fait l'objet d'essais d'analyse montrant que les thèmes des divers colloques ont été influencés tantôt par des principes théologiques et doctrinaux, tantôt par des questions œcuméniques, toujours en lien avec la liturgie, tantôt par l'actualité. À partir d'appréciations existantes, on mentionnera ci-dessous quelques exemples de thèmes d'exposés pris parmi beaucoup d'autres, brièvement présentés dans les limites de cette contribution.

Le Père Achille Triacca, fidèle participant des Semaines liturgiques depuis 1973 jusqu'à son décès en 2002, a aussi assuré la publication de leurs Actes durant ces années. On lui doit une analyse rétrospective parue en 1994, juste après le $4 \mathrm{O}^{\mathrm{e}}$ anniversaire de ces rencontres ${ }^{13}$. Il propose en particulier une énumération raisonnée du choix des thèmes annuels, qu'il s'efforce d'expliquer par des imbrications réciproques, selon lesquelles un domaine de recherche découle de celui de l'année précédente ; on trouve ainsi plusieurs préoccupations autour du thème de l'Église (thème de 1975, parution 1976 ; l'assemblée liturgique en 1976, parution 1977, puis un peu plus tard : L'Église dans la liturgie, 1979, parution 1980). Ont été ensuite examinées plusieurs actions sacramentelles : mariage, Eucharistie, ordinations (de 1993 à 1995, et parution l'année suivante à chaque fois). Le choix de ces intitulés pour les divers colloques annuels est aussi influencé par l'actualité œcuménique et les orientations données à divers dialogues ${ }^{14}$.

À titre d'exemples complémentaires, on peut encore mentionner le thème du jeûne, retenu voici quelques années en raison d'un impact médiatique donné à cette pratique en contexte chrétien ou autre ${ }^{15}$. L'exemple du $63^{\mathrm{e}}$ colloque, consacré en 2016 aux Traditions recomposées ${ }^{16}$ (parution 2017), est un choix résultant d'une prise de conscience de la différence nécessaire à souligner entre une tradition imposée de l'extérieur, la recomposition étant

13 P. A. Triacca, Le « Conferenze San Sergio » (supra, n. 9), p. 19-26.

14 Parcours à compléter par : P. M. Sodi, art. cité (supra, n. 7), p. 312-313, caractérisant diverses orientations thématiques et proposant des critères de choix appliqués au cours des divers colloques passés.

15 Parution: Jeûne et pratiques de repentance : dimensions communautaires et liturgiques. $58^{\mathrm{e}}$ Semaine d'Études liturgiques, Paris, Institut Saint-Serge, 27-30 juin 2011, Münster 2015 (Studia Oecumenica Friburgensia, 64).

16 Parution: réf. supra, n. 11. 
alors analysée comme artificielle, et l'authentique message théologique véhiculé par la liturgie qui non seulement le proclame, mais le met en œuvre. Une liturgie qui ne transforme pas ses participants en profondeur risque d'être réduite à un ritualisme. Ce constat fut d'ailleurs aussi l'un des acquis du $64^{\mathrm{e}}$ colloque, tenu en $2017^{17}$.

\subsection{Eléments constants : l'étude de sources}

Quel que soit le domaine choisi pour un colloque d'une année donnée, l'analyse de sources demeure aux yeux des organisateurs, une méthode de travail incontournable, pour éviter des considérations subjectives ou trop générales. Selon les orientations des divers spécialistes, il s'agit avant tout de sources liturgiques, mais l'examen de documents bibliques ou patristiques a aussi fourni l'occasion de contributions stimulantes ${ }^{18}$. La liturgie ne saurait se concevoir sans une prise en compte d'autres grands domaines de la théologie.

Au fil des exposés publiés dans les divers recueils, on trouve parfois formulés des éléments de méthode, parmi lesquels on peut relever ci-dessous un exemple à considérer comme représentatif de la manière dont les liturgistes souhaitent travailler à Saint-Serge. Dans l'une de ses nombreuses

17 Parution : Liturgie et religiosité. 64 e Semaine d'Études liturgiques, Paris, Institut Saint-Serge, 26-29 juin 2017, éd. A. Lossky, G. Sekulovski, T. Pott, Münster 2018 (Studia Oecumenica Friburgensia, 86).

18 Exemples d'exposés sur des thèmes bibliques : Y.-M. Blanchard, Bible et liturgie, dans : La liturgie, interprète de l'écriture II. Dans les compositions liturgiques, prières et chants. Conférences Saint-Serge. XLIXe Semaine d'Etudes liturgiques, Paris, 24-27 juin 2002, éd. C. Braga, A. Pistoia, Rome 2003, p. 259-276, sp. 265-269 (Bibliotheca Ephemerides Liturgicae. Subsidia, 126) ; F. Orfeuil, Peut-on parler d'homélies dans l'Ancien Testament?, dans : Nos pratiques homilétiques. Enjeux liturgiques et théologiques. $62^{\mathrm{e}}$ Semaine d'études liturgiques, Paris, Institut Saint-Serge, 22-25 juin 2015, éd. A. Lossky, G. Sekulovski, Münster 2017, p. 27-35, sp. 34 (Studia Oecumenica Friburgensia, 77) ; S. Caneri, De Hoshana rabba au Dimanche des Palmes l'attente ardente du Salut, dans : Liturgie et religiosité... (supra, n. 16), p. 47-62, sp. 54-56; thèmes patristiques : G. Sekulovski, Le recours aux Pères de l'Église dans les Semaines liturgiques, dans : 60 Semaines liturgiques... (supra, n. 6), p. 171-187, sp. 172-175 ; G. Sekulovski, Origène homme d'Église et prédicateur, dans : Nos pratiques homilétiques..., p. 37-51, sp. 46-50. 
contributions, le P. Charles Renoux insiste sur la nécessité de ne pas énoncer, en histoire de la liturgie, d'hypothèses hasardeuses ou mal fondées.

Participant régulier et assidu, actuellement le plus ancien des Semaines liturgiques, le P. Renoux est un connaisseur réputé de la liturgie ancienne de Jérusalem, dont on lui doit la mise au jour et l'analyse de sources à partir des $4^{\mathrm{e}}$ et $5^{\mathrm{e}}$ siècles. Ses nombreux travaux montrent que la liturgie de Jérusalem a influencé anciennement celles développées en d'autres régions : systèmes de lectures bibliques, fêtes et solennités, année liturgique ${ }^{19}$.

Son exposé intitulé « Jérusalem à Saint-Serge: histoire et hypothèses » fut prononcé lors du $60^{\mathrm{e}}$ colloque tenu en $2013^{20}$. Le propos s'attache à montrer, analyses documentaires à l'appui, que dans l'histoire des Églises, à une époque aussi reculée que celle de l'Itinéraire d'Égérie, d'autres régions que Jérusalem ne peuvent prétendre à une liturgie aussi élaborée, ce qui rend difficile l'hypothèse d'une influence extérieure sur la liturgie hagiopolite. De cette observation, l'auteur tire cette leçon méthodologique : une hypothèse est valable si des indices permettent de l'étayer ; selon lui : « une hypothèse... doit être en mesure de s'harmoniser avec les certitudes déjà apportées par d'autres écrits »; ces indices et ces certitudes sont à rechercher dans les documents qui nous sont effectivement parvenus ${ }^{21}$.

\section{7. Évolutions récentes}

Si le travail sur les sources demeure, aux yeux des organisateurs et des participants, une priorité, il vaut la peine de relever aussi quelques modifications dont l'avenir déterminera la pertinence. Certains exposés présentent des résultats de recherches scientifiques menées par des auteurs qui

19 L'abondante production scientifique de cet auteur montre l'influence de la liturgie de Jérusalem sur plusieurs autres traditions locales, notamment arménienne et géorgienne. Voir une liste de ses parutions dans les mélanges qui lui ont été offerts : Bibliographie Charles Athanase Renoux (O.S.B.,), dans : Sion, mère des Églises. Mélanges liturgiques offerts au Père Charles-Athanase Renoux, éd. M. D. Findikyan, D. Galadza, A. Lossky, Münster 2016, p. 19-34 (Semaines d'Études Liturgiques Saint-Serge. Supplément, 1).

20 Ch.-A. Renoux, Jérusalem à Saint-Serge : histoire et hypothèses, dans: 6o semaines liturgiques à Saint-Serge... (voir supra, n. 6), p. 211-240, sp. 236-239.

21 Ch.-A. Renoux, Jérusalem à Saint-Serge : histoire et hypothèses, p. 238. 
exercent aussi des responsabilités pastorales. Cela transparaît notamment dans leurs conclusions, qui proposent parfois brièvement quelques pistes susceptibles d'inspirer une catéchèse ou une prédication ; des conclusions peuvent encore influencer simplement l'esprit dans lequel un membre d'une assemblée envisage une action liturgique ${ }^{22}$.

Sans confondre le domaine liturgique avec les questions pastorales, ces dernières peuvent aussi faire l'objet d'une investigation scientifique, dans la mesure où certaines d'entre elles sont liées à la liturgie. Tel a été le constat fait par les organisateurs, qui ont ainsi pris la décision, voici quelques années, d'ouvrir également et de manière raisonnée les travaux des colloques liturgiques de Saint-Serge à des réalités de terrain. Ainsi par exemple, l'étude du P. Marcel Metzger dans le volume déjà mentionné, consacré en 2011 au thème du jeûne, exploite des coupures de presse et des annonces de Carême, en comparaison avec le battage médiatique fait autour de pratiques de restrictions alimentaires, chrétiennes ou non ${ }^{23}$. Cette contribution constitue un bon exemple de la manière d'analyser des réalités liturgiques d'aujourd'hui : pour que ces observations puissent profiter à d'autres, il est indispensable, comme cela est fait dans l'exemple cité, d'analyser des données provenant de l'actualité du moment. Les observations et analyses de réalités pastorales obéissent à leur méthodologie propre, à ne pas confondre avec celle applicable aux faits liturgiques.

Une autre évolution récente, dans l'organisation des Semaines liturgiques à Saint-Serge, concerne le public invité à proposer une contribution. Il a été décidé, depuis le $63^{\mathrm{e}}$ colloque tenu en $2016^{24}$, d'ouvrir la participation, avec exposé, à de jeunes chercheurs (niveau minimum : Master), pour offrir

22 Exemples: contributions des P. N. Cernokrak ou E. Cothenet lors des colloques de 1988 ou 1992, parus en 1989 et 1993 ; références : A. Triacca, Le "Conferenze San Sergio » (supra, n. 9), p. 104 et 112. Plus récemment: M. Stavrou, Un renouveau liturgique orthodoxe en france au $\mathrm{XX}$ e siècle, signe de la réception de quelques principes théologiques, dans : Liturges et liturgistes. Fructification de leurs apports dans l'aujourd'hui des Églises. $59^{\mathrm{e}}$ Semaine d'Études Liturgiques, Paris, Institut Saint-Serge, 25-28 juin 2012, Münster 2015, p. 11-29, ici 22-28 (Studia Oecumenica Friburgensia, 69).

23 M. Metzger, Entretenir sa forme : laquelle? Restrictions alimentaires et religions, dans : Jeûne et pratiques de repentance... (supra, n. 14), p. 9-26, sp. 11-19.

24 Voir supra, n. 11. 
aux doctorants la possibilité de présenter leurs recherches faites ou en cours. Cet élargissement, dont le bien-fondé reste à évaluer, offre tout au moins un double avantage. Il permet aux liturgistes plus anciens de repérer les tendances et les centres d'intérêt des nouvelles recherches; il offre aussi la possibilité aux jeunes chercheurs de voir leur travail publié, en certains cas une première fois. Ainsi, la présentation orale d'un exposé discuté en salle ne vaut plus approbation éditoriale, ni admission automatique en publication, mais celle-ci dépend finalement d'une expertise scientifique du texte envoyé ensuite par l'auteur. À un aspect scientifique, toujours recherché dans ces colloques liturgiques, s'ajoute depuis peu une dimension pédagogique.

\section{Travaux d'édition et impact possible des Semaines Saint- -Serge : conclusion}

Il paraît finalement important de préciser les modalités de publication des travaux. Selon les évaluations déjà mentionnées des Pères A. Triacca, puis M. Sodi, les travaux liturgiques de Saint-Serge ont été publiés régulièrement à partir de 1965, d'abord dans la collection Lex Orandi (éditions du Cerf), puis successivement chez d'autres éditeurs ${ }^{25}$.

Durant 3 ans, de 2011 à 2013, pour des raisons à la fois institutionnelles et financières, les Semaines liturgiques ont été organisées à Saint-Serge sans garantie de publication prochaine des travaux, ce qui frisait l'asphyxie scientifique. Organiser un colloque sans en publier ensuite les Actes constitue un danger d'errance et de perte de rigueur, affaiblissant la crédibilité des propos tenus. Grâce à l'intervention de plusieurs chercheurs amis de l'Institut Saint-Serge, et des Semaines liturgiques en particulier, parmi lesquels le pasteur Bruno Buerki, membre et ancien président de la Societas Liturgica, et le professeur Heinzgerd Brakmann, membre de la Society of Oriental Liturgy, une aide financière provenant de l'Université de Fribourg $(\mathrm{CH})$ a pu être octroyée pour faire paraître les Actes dans une collection, spécialement constituée aux éditions Aschendorff à Münster (Allemagne).

25 Récapitulation la plus récente : P. M. Sodi, art. cité (supra, n. 7), p. 313-315; voir aussi P. M. Sodi, art. cité (supra, n. 8). 
Les actes des Semaines liturgiques Saint-Serge sont également intégrés dans la série Studia Oecumenica Friburgiensia, ce qui leur procure un signalement plus large.

Depuis la parution des Actes à partir de 2011 dans cette nouvelle série, le retard a été comblé et le volume du colloque de 2017 a paru en 2018 . Sous réserve que les intervenants respectent les exigences techniques éditoriales et les délais d'envoi des textes, le volume regroupant les contributions peut paraître dans l'année qui suit le colloque. La mise au point technique est assurée entre autres par une équipe de relecteurs et correcteurs bénévoles.

Les points de vue exprimés dans les articles sont publiés sous l'unique responsabilité de leurs auteurs ; les Semaines d'études liturgiques Saint-Serge ne déterminent pas d'autre ligne éditoriale que l'exigence d'un niveau scientifique attendu dans les contributions. Les membres du Comité scientifique veillent à maintenir cette qualité, se réservant le droit de ne pas admettre une contribution dans un volume, si le niveau en est jugé insuffisant, indépendamment des idées exprimées.

Concernant l'impact des Semaines liturgiques St-Serge, il est difficile d'en évaluer l'influence, en l'absence de statistiques de ventes des publications. Mais lors du $60^{\mathrm{e}}$ colloque tenu en 2013, une communication à deux voix, proposée par le Père Serge Sollogoub et son épouse Anne, a jeté quelque lumière sur cet impact. Les auteurs font état de certaines contributions qui ont pu influencer l'esprit théologique dans lequel il convient d'envisager certaines célébrations liturgiques ${ }^{26}$.

Enfin, on peut signaler la parution de recensions de quelques volumes dans des périodiques liturgiques tels que par exemple " Ecclesia orans », « Ephemerides liturgicae », « Proche-Orient chrétien », " La Maison-Dieu », la « Revue théologique de Louvain ». On y trouve salués la teneur scien-

26 S. et A. Sollogoub, L'apport des Conférences Saint-Serge aux différentes familles liturgiques: un point de vue orthodoxe, dans: Les mouvements liturgiques. Corrélations entre pratiques et recherches. Conférences Saint-Serge. Le Semaine d'Études liturgiques, Paris, 23-26 juin 2003, éd. C. Braga, A. Pistoia, Rome 2004 (Bibliotheca Ephemerides Liturgicae. Subsidia, 129), p. 301-308, dimension œcuménique : p. 303-304; exemple d'impact dans l'action pastorale : p. 306. Voir aussi, plus récemment: M. Metzger, L'étude de la liturgie, comme théologie pastorale, dans les semaines d'études liturgiques de Saint-Serge, dans : Liturges et liturgistes... (supra, n. 21), p.349-363, caractérisation d'une théologie pastorale découlant de la liturgie : p. 358-361. 
tifique des exposés ainsi que la tenacité des organisateurs comme des contributeurs, dont les efforts maintiennent régulièrement ces colloques depuis longtemps, sous une forme récemment renouvelée.

Mais les dernières décisions d'ouverture à un public restant universitaire et à des champs plus larges doivent encore faire leurs preuves : la diffusion internationale des travaux permettra d'évaluer l'opportunité de ces nouvelles orientations. Dans les parutions récentes, des recenseurs ont aussi relevé quelques points à parfaire, notamment une certaine hétérogénéité thématique parmi les exposés regroupés en un même volume, et aussi une inégalité quant au niveau scientifique de plusieurs exposés, souvent signés par de jeunes chercheurs qui doivent encore faire leurs preuves. Un tel constat incite les organisateurs et le comité éditorial à une vigilance accrue : ainsi, un exposé présenté oralement dans un colloque sera désormais systématiquement soumis à une expertise scientifique avant admission à publication.

Finalement, c'est la diffusion internationale des travaux qui permettra d'évaluer l'opportunité de ces nouvelles orientations, prises en vue d'une plus grande rigueur dans l'étude des faits liturgiques. Les organisateurs espèrent ainsi améliorer encore la qualité de ces colloques, en vue de mieux discerner ce qui favorisera des liturgies plus vivantes, appelées à assurer toujours mieux la fonction actualisatrice de tout culte chrétien.

\section{Streszczenie}

Tygodnie liturgiczne św. Sergiusza (Saint Serge) w Paryżu i ich wkład w odnowe liturgiczna

Założony w Paryżu w 1925 roku przez prawosławnych emigrantów rosyjskich Instytut Teologii Prawosławnej Świętego Sergiusza, który na co dzień funkcjonuje także jako ośrodek kultu (cerkiew), jest ośrodkiem myśli teologicznej, wykładów i badań, w których szczególne miejsce zajmuje problematyka liturgiczna. Po krótkim ukazaniu historii Instytutu, jego powstania i znaczenia dla Francji i Europy Zachodniej referat analizuje ducha, w jakim organizowane są w tymże Instytucie konferencje liturgiczne (Tygodnie Studiów Liturgicznych), które są spotkaniami o charakterze ekumenicznym i naukowym, skoncentrowanymi wokół problematyki 
316 André Lossky

(tematyki) liturgicznej. Przedstawione są stałe elementy konferencji, wśród których ważne miejsce zajmuje badanie źródeł liturgicznych, a także kilka ostatnich nowych propozycji metodologicznych, które poddawane są ocenie badaczy i duszpasterzy zatroskanych o przyszłość liturgii chrześcijańskiej. 